Noname manuscript No.

(will be inserted by the editor)

\title{
Establishing Nash Equilibrium of the Manufacturer- Supplier Game in Supply Chain Management
}

\author{
James Ang • Masao Fukushima • Fanwen \\ Meng - Takahiro Noda • Jie Sun
}

Received: / Accepted:

\begin{abstract}
We study a multi-leader, one-follower game model in supply chain optimization where $n$ suppliers compete to provide a single product for a manufacturer. We regard the selling price of each supplier as a pre-determined parameter and consider the case that suppliers compete on the basis of delivery frequency to the manufacturer. Each supplier's profit depends not only on its own delivery frequency, but also on other suppliers' frequencies through their impact on manufacturer's purchase allocation to the suppliers. We first solve the follower's (manufacturer's) purchase allocation problem by deducing an explicit formula of its solution. We then formulate the $n$ leaders' (suppliers') game as a generalized Nash game with shared constraints, which is theoretically difficult, but in our case could be solved numerically by converting to a regular variational inequality problem. For the special case that the selling prices of all suppliers are identical, we provide a sufficient and necessary condition for the existence
\end{abstract}

This paper was presented at The Eighth International Conference on Optimization: Techniques and Applications (ICOTA8) in Shanghai, December 2010.

James Ang

Department of Decision Sciences, National University of Singapore. E-mail: bizangsk@nus.edu.sg

Masao Fukushima

Department of Applied Mathematics and Physics, Graduate School of Informatics, Kyoto University. E-mail: fuku@i.kyoto-u.ac.jp

Fanwen Meng

Centre for Maritime Studies, National University of Singapore. E-mail: cmsmf@nus.edu.sg

Takahiro Noda

Department of Applied Mathematics and Physics, Graduate School of Informatics, Kyoto University. E-mail: noda@amp.i.kyoto-u.ac.jp

Jie Sun

Department of Decision Sciences and Risk Management Institute, National University of Singapore. Tel.: +65-6516-6448 Fax: +65-6779-2621 E-mail: jsun@nus.edu.sg 
and uniqueness of the Nash equilibrium. An explicit formula of the Nash equilibrium is obtained and its local uniqueness property is proved.

Keywords Supply Chain Management - Leader-Follower Game · Nash Equilibrium . Nonlinear Programming

\section{Introduction}

Variational inequalities (VI) provide an avenue for addressing equilibrium conditions as a compact form for Nash games with continuous-strategies. When the games are generalized to allow mutually dependent strategy sets, the resulting games are referred to as generalized Nash games or generalized Nash equilibrium problems, of which variational conditions may be represented as quasi-variational inequalities (QVI). In particular, when all players in a game share common constraints, such a game is called a generalized Nash game with shared constraints. Let $\mathcal{N}$ be a set of players and $N=|\mathcal{N}|$. Let $x_{i} \in \Re^{m_{i}}$ and $\vartheta_{i}$ be the strategy and the objective function with respect to player $i \in \mathcal{N}$. The generalized Nash game with shared constraints is defined as follows. For each $i \in \mathcal{N}$, given $x_{-i}$, player $i$ seeks to solve the following parameterized optimization problem for $x_{i}$ :

$$
\begin{array}{ll}
\min _{x_{i}} & \vartheta\left(x_{i} ; x_{-i}\right) \\
\text { s. t. } & x_{i} \in K_{i}\left(x_{-i}\right),
\end{array}
$$

where $x_{-i}:=\left(x_{1}, \ldots, x_{i-1}, x_{i+1}, \ldots, x_{N}\right)^{T}, K_{i}\left(x_{-i}\right):=\left\{z \in \Re^{m_{i}} \mid\left(z ; x_{-i}\right) \in S\right\}$ with $\left(z ; x_{-i}\right):=\left(x_{1}, \ldots, x_{i-1}, z, x_{i+1}, \ldots, x_{N}\right)^{T}$. Here $T$ stands for the transpose and $S \subseteq \Re^{\sum_{i=1}^{N} m_{i}}$ denotes the feasible set defined by the shared constraints. Further, a strategy tuple $x=\left(x_{1}, \ldots, x_{N}\right)^{T}$ is called a generalized Nash equilibrium (GNE) of this game if, for each $i \in \mathcal{N}, x_{i}$ is an optimal solution of the above problem.

The early study of GNEs dates back at least to Debreu [6] and Arrow and Debreu [1], where a generalized Nash game was called a social equilibrium (problem) or an abstract economy. The generalized Nash game with shared constraints was studied by Rosen [18]. In recent years, generalized Nash games have been intensively studied, for instance, see $[11,17,7]$ and the references therein. In particular, the Nash equilibrium has been investigated in various fields of economics, engineering science, and management science. Motivated by such an application in supply chain management, we investigate a specific class of generalized Nash game in this paper. Specifically, we consider a supply chain network consisting of a single manufacturer and a number of suppliers where the manufacturer has to accommodate orders emanating from different locations and at different times under uncertain demand from the customers. Due to the large number of potential suppliers, each supplier has little bargain power on price. Instead, they would usually bid on the capability to deliver within a certain time window. In short, suppliers should ship as often as possible without the associated cost increase due to expediting or relying on more expensive modes of transport. The manufacturer, on the other hand, has to decide on the amount to allocate to each supplier as it does not want a deadlock situation where all the stocks arrive at the factory at the same time. Put simply, for a fixed price, the manufacturer may have to choose a set of suppliers who can ship more frequently. While many papers have discussed the "management" side of competition where delivery speed is treated as a source of competitive 
advantage, as we see e.g., from $[9,12,13,16,14,19,10,4,3,2,5]$, our focus in this paper is to address the "optimization" side of this problem; namely, we are concerned with the general mathematical structure of the problem, possible global optimal solutions and the uniqueness of the solution.

The analysis of this paper is basically based on the framework of variational inequalities and the leader-follower game theory. For a general survey of stochastic optimiza-

tion problems with equilibrium constraints, see the recent work by Lin and Fukushima [15]. The contributions of this paper are as follows.

1. We extend the 2 person-game as studied in the literature to $n$-leaders and 1 -follower game. We show that the order allocation problem of the follower is a convex separable quadratic programming problem and its solution formula is derived.

2. We demonstrate that the supplier-competition model can be reformulated as a generalized Nash game with shared constraints, in which each player's feasible set depends on other players' decisions.

3. While this game is theoretically difficult to deal with, our third contribution is that, for the special case where all suppliers offer the same selling price, we show that an all positive Nash equilibrium exists and it can be computed by solving a quadratic equation system.

4. Under the same setting, we also derive an explicit formula for the unique Nash equilibrium in the leaders' game and provide necessary and/or sufficient conditions for the existence and uniqueness of such a solution.

The rest of this paper is organized as follows. Following the introduction, Section 2 provides a formulation of supplier competition based on delivery frequency with predetermined price and demand uncertainty. Section 3 investigates the Nash equilibrium of the delivery competition game. A sufficient condition for the existence of a nontrivial Nash equilibrium is established. Section 4 discusses the special case where all suppliers sell their products at the same price, followed by some management insights. Section 5 concludes the paper.

\section{Model Development of the Multi-leader Single-follower Game}

In this section, we consider the situation where the suppliers are the leaders and the manufacturer is the follower. This is a bi-level or two-stage problem, in which the suppliers of the upper level problem will compete on delivery frequency, depending on the follower's action of the lower-level problem associated with the manufacturer. We shall establish this model and then investigate its properties of interest. First, we list the notations on the variables and parameters related to all players involved in the supply chain. For $i=1,2, \ldots, n$ :

Variables:

$r_{i}$ : expected delivery frequency of supplier $i$;

$\lambda_{i}$ : demand allocations to each supplier $i$ determined by the manufacturer.

Parameters:

D: the manufacturer's random demand which follows a certain distribution;

$\mathbb{E}[\mathbf{D}]$ : mathematical expectation of $\mathbf{D}$;

$h$ : unit inventory holding cost of the manufacturer; 
$l_{i}$ : lower bound of the expected delivery frequency of supplier $i$;

$u_{i}$ : upper bound of the expected delivery frequency of supplier $i$;

$p_{i}$ : unit selling price of the product set by supplier $i$;

$c_{i}$ : unit cost of production of supplier $i$;

$C_{i}$ : fixed cost of production of supplier $i$;

$k_{i}$ : unit cost of delivery of supplier $i$;

$K_{i}$ : fixed cost of delivery of supplier $i$.

In the game presented below, $r_{i}, i=1, \ldots, n$, are decision variables determined by suppliers, while $\lambda_{i}, i=1, \ldots, n$, are decision variables determined by the manufacturer. In the following analysis, we assume that the parameters $h>0, u_{i} \geq l_{i}>0, p_{i}>0$, $c_{i}>0, C_{i}>0, k_{i}>0$, and $K_{i}>0$ are fixed scalars. For simplicity of discussion, we consider the expectation of the manufacturer's demand, $\mathbb{E}[\mathbf{D}]>0$, throughout this paper.

In the problem under consideration, we assume that suppliers may set different prices $p_{i}$ of the product and those prices are fixed constants. Moreover, we assume that the selling price $p_{i}$ of the product is greater than the sum of unit production $\operatorname{cost} c_{i}$ and unit transportation cost $k_{i}$ of the product, i.e., $p_{i}>c_{i}+k_{i}$, for each supplier $i$.

As to the variables, each supplier $i$ has to make the decision $r_{i}$ on delivery frequency of the product, while the respective demand allocations $\lambda_{i}$ are determined by the manufacturer based on the prices $p_{i}$ and delivery frequencies $r_{i}$ given by all suppliers. Since each $\lambda_{i}$ represents a fraction or a portion of the total demand assigned to supplier $i$, they satisfy $\sum_{i=1}^{n} \lambda_{i}=1, \lambda_{i} \geq 0, i=1, \ldots, n$. The objective of each supplier is to maximize his profit, while the manufacturer aims to minimize the total cost incurred. The suppliers compete each other on delivery frequencies in order to obtain the share of demand allocation as much as possible from the manufacturer, thereby achieving their maximum profits.

We formulate this problem as a bi-level non-cooperative game; in the upper level, the suppliers compete on delivery frequencies without adopting any cooperative strategy or share any information among them, while in the lower level, the manufacturer determines the demand allocations to the suppliers in response to the delivery frequencies given by the suppliers.

Let $\Phi_{i}(r, \lambda)$ denote the expected profit function of each supplier $i(i=1, \ldots, n)$ and $\Psi(\lambda, r)$ the expected cost function of the manufacturer, where $\lambda=\left(\lambda_{1}, \ldots, \lambda_{n}\right)^{T}$ and $r=\left(r_{1}, \ldots, r_{n}\right)^{T}$. Then it follows that

$$
\Phi_{i}(r, \lambda) \equiv\left(p_{i}-c_{i}-k_{i}\right) \lambda_{i} \mathbb{E}[\mathbf{D}]-\left(C_{i}+K_{i}\right) r_{i}
$$

In addition, using the EOQ logic, it is known that the total purchase cost together with the inventory cost of the manufacturer is as follows:

$$
\Psi(\lambda, r) \equiv \sum_{i=1}^{n}\left(p_{i} \lambda_{i}+\frac{h \lambda_{i}^{2}}{2 r_{i}}\right) \mathbb{E}[\mathbf{D}]
$$

The mathematical model of the underlying game is therefore stated as follows. Each leader (supplier) solves the following problems for $r_{i}$ :

$$
\max _{r_{i}} \Phi_{i}(r, \lambda) \quad \text { s.t. } \quad l_{i} \leq r_{i} \leq u_{i},
$$


while the follower (manufacturer) solves the following problem for $\lambda$ :

$$
\min _{\lambda} \Psi(\lambda, r) \quad \text { s.t. } \quad \sum_{i=1}^{n} \lambda_{i}=1, \lambda_{i} \geq 0, i=1, \ldots, n .
$$

This is a bi-level game. Apparently, the objectives of the upper-level problems (the leaders' problems) are of implicit form since $\lambda$ is an optimal solution of the lower-level problem (the follower's problem) in which $r$ is a parameter. Note also that the objective function of the lower-level problem is strictly convex and quadratic, hence the optimal solution, denoted by $\lambda(r)$, exists and is unique. In other words, an optimal demand allocation $\lambda(r)$ by the manufacturer is uniquely determined in response to delivery frequencies $r$ of the suppliers. Thus, the underlying bi-level model can be rewritten as

$$
\max _{r_{i}} \Phi_{i}(r, \lambda(r)) \quad \text { s.t. } \quad l_{i} \leq r_{i} \leq u_{i}, \text { and } \lambda(r) \text { is the solution to }(4)
$$

Now let us derive the optimal solution $\lambda(r)$ of the manufacturer's problem (4). Since the problem is strictly convex and feasible, it is necessary and sufficient that the optimal solution $\lambda$ along with Lagrange multipliers $v$ and $w=\left(w_{1}, \ldots, w_{n}\right)^{T}$ satisfies the following Karush-Kuhn-Tucker (KKT) conditions:

$$
\begin{aligned}
& p_{i}+\frac{h}{r_{i}} \lambda_{i}-v-w_{i}=0 \quad i=1, \ldots, n, \\
& \sum_{i=1}^{n} \lambda_{i}=1 \\
& \lambda_{i} \geq 0, w_{i} \geq 0, \lambda_{i} w_{i}=0 \quad i=1, \ldots, n .
\end{aligned}
$$

From (6) and (8), we have

$$
w_{i}=p_{i}+\frac{\lambda_{i}}{r_{i}} h-v \geq 0, \quad i=1, \ldots, n,
$$

which implies

$$
\lambda_{i} \geq \frac{r_{i}}{h}\left(v-p_{i}\right), \quad i=1, \ldots, n .
$$

Moreover, taking into account the complementarity condition (8), we obtain

$$
\lambda_{i}=\max \left(0, \frac{r_{i}}{h}\left(v-p_{i}\right)\right), \quad i=1, \ldots, n .
$$

It then follows from (7) that

$$
\sum_{i=1}^{n} \max \left(0, \frac{r_{i}}{h}\left(v-p_{i}\right)\right)=1 .
$$

Let $v^{*}$ denote the unique solution of this equation. Then the optimal solution $\lambda^{*}=$ $\left(\lambda_{1}^{*}, \ldots, \lambda_{n}^{*}\right)^{T}$ to problem (4) is given by

$$
\lambda_{i}^{*}=\max \left(0, \frac{r_{i}}{h}\left(v^{*}-p_{i}\right)\right), \quad i=1, \ldots, n .
$$

It is reasonable to require $v^{*}>p_{i}$ for $i=1, \ldots, n$ for otherwise we should instead consider $k(<n)$ leaders rather than the $n$ leaders by ignoring the suppliers with zero allocation. This requirement imposes a set of new constraints among the leaders as 
follows. From (9) and $v^{*}>p_{i}$, one has $v^{*}=\left(\sum_{j=1}^{n} r_{j}\right)^{-1}\left(\sum_{j=1}^{n} r_{j} p_{j}+h\right)$. Substituting it into (10), we have

$$
\lambda_{i}(r)=\frac{r_{i}}{\sum_{j=1}^{n} r_{j}}\left[1+\frac{1}{h} \sum_{j=1}^{n} r_{j}\left(p_{j}-p_{i}\right)\right], \quad i=1, \ldots, n .
$$

Thus the condition $v^{*}>p_{i}$ (i.e. $\lambda_{i}(r)>0$ ) for $i=1, \ldots, n$ is equivalent to that there exists a positive number $\varepsilon$ such that

$$
\sum_{j=1}^{n} r_{j}\left(p_{i}-p_{j}\right) \leq h-\varepsilon, \quad i=1, \ldots, n
$$

which is a set of shared constraints for the upper level $n$-leaders game.

By substituting the optimal response $\lambda(r)$ given by (11) into the function $\Phi_{i}$ defined by (1), the objective function of supplier $i$ becomes

$$
\Phi_{i}(r, \lambda(r))=\frac{\left(p_{i}-c_{i}-k_{i}\right) \mathbb{E}(\mathbf{D}) r_{i}}{\sum_{j=1}^{n} r_{j}}\left[1+\frac{1}{h} \sum_{j=1}^{n} r_{j}\left(p_{j}-p_{i}\right)\right]-\left(C_{i}+K_{i}\right) r_{i} .
$$

For ease of notation, let

$$
\alpha_{i} \equiv \frac{\left(p_{i}-c_{i}-k_{i}\right) \mathbb{E}(\mathbf{D})}{C_{i}+K_{i}}, \quad i=1, \ldots, n .
$$

Note that the assumption $p_{i}>c_{i}+k_{i}$ made earlier in this section ensures $\alpha_{i}>0$ for all $i=1, \ldots, n$. Moreover, define the functions $\phi_{i}(r)$ by

$$
\phi_{i}(r) \equiv \frac{1}{C_{i}+K_{i}} \Phi_{i}(r, \lambda(r))=\frac{\alpha_{i} r_{i}}{\sum_{j=1}^{n} r_{j}}\left[1+\frac{1}{h} \sum_{j=1}^{n} r_{j}\left(p_{j}-p_{i}\right)\right]-r_{i}, \quad i=1, \ldots, n .
$$

Since $C_{i}+K_{i}>0$ for all $i$, in terms of finding an equilibrium point of the leaders' game, we could instead consider $\phi_{i}(r)$ as the actual objective function of player $i$. Then, taking into account the constraint (12), the optimization problem (5) of supplier $i$ in the upper-level can be rewritten as

$$
\max _{r_{i}} \phi_{i}\left(r_{i}, r_{-i}\right) \quad \text { s.t. } \quad \sum_{j=1}^{n} r_{j}\left(p_{i}-p_{j}\right) \leq h-\varepsilon, l_{i} \leq r_{i} \leq u_{i},
$$

where $r_{-i}=\left(r_{1}, \ldots, r_{i-1}, r_{i+1}, \ldots, r_{n}\right)^{T}$ is given as exogenous. This is a generalized Nash game, since the constraints in (14) contain the other suppliers' variables $r_{-i}$. We denote the feasible set of problem (14) by $\mathcal{S}_{i}\left(r_{-i}\right)$. Moreover, let us define the set

$$
\mathcal{S} \equiv\left\{r=\left(r_{1}, \ldots, r_{n}\right)^{T} \mid \sum_{j=1}^{n} r_{j}\left(p_{i}-p_{j}\right) \leq h-\varepsilon, l_{i} \leq r_{i} \leq u_{i}, i=1, \ldots, n\right\} .
$$

Then it is easy to see the following relation:

$$
r \in \mathcal{S} \Longleftrightarrow r_{i} \in \mathcal{S}_{i}\left(r_{-i}\right), \quad i=1, \ldots, n
$$

In other words, the feasible sets of all leaders' problems are defined by means of the set $\mathcal{S}$, that is, all leaders share the common constraints. 
Note that problem (14) is particularly a generalized Nash game with shared constraints. Recall that a vector $r^{*}=\left(r_{1}^{*}, \ldots, r_{n}^{*}\right)^{T}$ is a generalized Nash equilibrium, if, for each $i=1, \ldots, n, r_{i}^{*}$ is an optimal solution of the optimization problem (14) with $r_{-i}$ fixed at $r_{-i}^{*}$. It is known that a generalized Nash game can be reformulated as a quasi-variational inequality problem (see Harker [11], Pang and Fukushima [17], for example), which is, however, generally difficult to solve. In the next section, we will concentrate on a special class of GNEs called a normalized equilibrium (see Rosen [18]) and discuss reformulation of a generalized Nash game with shared constraints as a variational inequality problem (see, e.g., Facchinei and Kanzow [7]) that is much easier to deal with than a quasi-variational inequality problem.

\section{Variational Inequality Formulation and Normalized Equilibrium}

First we remark that the set of inequalities $\sum_{j=1}^{n} r_{j}\left(p_{i}-p_{j}\right) \leq h-\varepsilon, i=1, \ldots, n$, can be replaced by the single inequality $\sum_{j=1}^{n} r_{j}\left(\bar{p}-p_{j}\right) \leq h-\varepsilon$, where $\bar{p}=\max \left(p_{1}, \ldots, p_{n}\right)$. Thus the set $\mathcal{S}$ is given by

$$
\mathcal{S}=\left\{r=\left(r_{1}, \ldots, r_{n}\right)^{T} \mid \sum_{j=1}^{n} r_{j}\left(\bar{p}-p_{j}\right) \leq h-\varepsilon, l_{i} \leq r_{i} \leq u_{i}, i=1, \ldots, n\right\} .
$$

Define the function $g(r)$ by

$$
g(r) \equiv \sum_{j=1}^{n} r_{j}\left(\bar{p}-p_{j}\right)-h+\varepsilon
$$

Then the supplier $i$ 's problem (14) can be rewritten as

$$
\max \phi_{i}\left(r_{i}, r_{-i}\right) \quad \text { s.t. } g\left(r_{i}, r_{-i}\right) \leq 0, l_{i} \leq r_{i} \leq u_{i}
$$

and the KKT conditions for this problem are given by

$$
\begin{aligned}
& \nabla_{r_{i}} \phi_{i}\left(r_{i}, r_{-i}\right)-\mu_{i} \nabla_{r_{i}} g\left(r_{i}, r_{-i}\right)-\xi_{i}+\eta_{i}=0, \\
& \mu_{i} \geq 0, g\left(r_{i}, r_{-i}\right) \leq 0, \mu_{i} g\left(r_{i}, r_{-i}\right)=0, \\
& \xi_{i} \geq 0, u_{i}-r_{i} \geq 0, \xi_{i}\left(u_{i}-r_{i}\right)=0, \\
& \eta_{i} \geq 0, r_{i}-l_{i} \geq 0, \eta_{i}\left(r_{i}-l_{i}\right)=0,
\end{aligned}
$$

where $\mu_{i}, \xi_{i}, \eta_{i}$ are Lagrange multipliers. Since each problem (16) is a convex programming problem with linear constraints, the KKT conditions (17) are necessary and sufficient optimality conditions. Therefore, a vector $r=\left(r_{1}, \ldots, r_{n}\right)^{T}$ is a GNE if and only if it along with some Lagrange multipliers $\xi_{1}, \ldots, \xi_{n}, \eta_{1}, \ldots, \eta_{n}$ and $\mu_{1}, \ldots, \mu_{n}$ satisfies the concatenated KKT system

$$
\left.\begin{array}{rl}
\nabla_{r_{i}} \phi_{i}\left(r_{i}, r_{-i}\right)-\mu_{i} \nabla_{r_{i}} g\left(r_{i}, r_{-i}\right)-\xi_{i}+\eta_{i}=0 \\
\mu_{i} \geq 0, g\left(r_{i}, r_{-i}\right) \leq 0, \mu_{i} g\left(r_{i}, r_{-i}\right)=0 \\
\xi_{i} \geq 0, u_{i}-r_{i} \geq 0, \xi_{i}\left(u_{i}-r_{i}\right)=0 \\
\eta_{i} \geq 0, r_{i}-l_{i} \geq 0, \eta_{i}\left(r_{i}-l_{i}\right)=0
\end{array}\right\} \quad i=1, \ldots, n
$$

Now let us consider the following variational inequality problem (VIP): Find a vector $r \in \mathcal{S}$ such that

$$
\left(r^{\prime}-r\right)^{T} F(r) \geq 0 \quad \forall r^{\prime} \in \mathcal{S},
$$


where $F(r)$ is the mapping defined by

$$
F(r) \equiv\left(\begin{array}{c}
-\nabla_{r_{1}} \phi_{1}(r) \\
\vdots \\
-\nabla_{r_{n}} \phi_{n}(r)
\end{array}\right) .
$$

Since the mapping $F(r)$ is continuous and the set $\mathcal{S}$ is nonempty, closed, convex and bounded, the VIP (19) has a solution. Since $\mathcal{S}$ is given by (15), a vector $r$ is a solution of the VIP (19) if and only if it along with some Lagrange multipliers $\xi_{1}, \ldots, \xi_{n}, \eta_{1}, \ldots, \eta_{n}$ and $\mu$ satisfies the following KKT conditions for the VIP (19):

$$
\left.\begin{array}{rl}
\nabla_{r_{i}} \phi_{i}\left(r_{i}, r_{-i}\right)-\mu_{i} \nabla_{r_{i}} g\left(r_{i}, r_{-i}\right)-\xi_{i}+\eta_{i}=0 \\
\mu \geq 0, g\left(r_{i}, r_{-i}\right) \leq 0, \mu g\left(r_{i}, r_{-i}\right)=0 \\
\xi_{i} \geq 0, u_{i}-r_{i} \geq 0, \xi_{i}\left(u_{i}-r_{i}\right)=0 \\
\eta_{i} \geq 0, r_{i}-l_{i} \geq 0, \eta_{i}\left(r_{i}-l_{i}\right)=0
\end{array}\right\} \quad i=1, \ldots, n
$$

Note that the only difference between the two KKT systems (18) and (21) is that (18) contains the Lagrange multipliers $\mu_{1}, \ldots, \mu_{n}$ corresponding to the respective shared constraints $g\left(r_{i}, r_{-i}\right) \leq 0$ in problems (16), while (21) contains only one Lagrange multiplier $\mu$ corresponding to the constraint $g(r) \leq 0$ in the VIP (19).

In fact, a GNE with Lagrange multipliers $\mu_{i}, i=1, \ldots, n$, satisfying (18) and the additional condition

$$
\mu_{1}=\mu_{2}=\cdots=\mu_{n}
$$

is a special GNE called a normalized equilibrium first studied by Rosen [18]. By direct comparison of the two KKT systems (18) and (21), it is easily seen that a normalized equilibrium can be obtained as a solution of the VIP (19).

Next we consider the uniqueness of a normalized equilibrium by way of the uniqueness of a solution to the VIP (19). From the well known result in variational inequality problems (see, e.g., Facchinei and Pang [8]), a solution of the VIP (19) is unique if the mapping $F(r)$ is strictly monotone. A sufficient condition for the strict monotonicity of the mapping $F(r)$ is that the Jacobian matrix $F^{\prime}(r)$ is positive definite for any $r$. Moreover, since an asymmetric square matrix $A$ is positive definite if and only if its symmetric part $A+A^{T}$ is positive definite, and any diagonally dominant matrix with positive diagonal elements is positive definite, we can deduce that a normalized equilibrium is unique if the matrix $F^{\prime}(r)+F^{\prime}(r)^{T}$ is diagonally dominant and its diagonal elements are all positive. By the definition $(20)$ of $F(r)$, its Jacobian $F^{\prime}(r)=\left(-\frac{\partial^{2} \phi_{i}(r)}{\partial r_{i} \partial r_{j}}\right)$, which we denote $A=\left(a_{i j}\right)$ for notational simplicity, is given by

$$
\begin{array}{rlr}
a_{i i}= & \frac{2 \alpha_{i}}{R^{3}}\left[1+\frac{1}{h} \sum_{k=1}^{n} r_{k}\left(p_{k}-p_{i}\right)\right]\left(R-r_{i}\right), & i=1, \ldots, n, \\
a_{i j}= & \frac{\alpha_{i}}{R^{3}}\left[1+\frac{1}{h}\left(\sum_{k=1}^{n} r_{k}\left(p_{k}-p_{i}\right)-r_{j}\left(p_{j}-p_{i}\right)\right)\right]\left(2 r_{i}-R\right) & \\
& -\frac{\alpha_{i}\left(p_{j}-p_{i}\right)}{h R^{3}}\left(2 r_{i} r_{j}+R^{2}-r_{j} R\right), & i, j=1, \ldots, n ; i \neq j,
\end{array}
$$

where $\alpha_{i}$ are given by (13) and $R \equiv \sum_{j=1}^{n} r_{j}$. Therefore a normalized equilibrium is unique if the following condition is satisfied for all $i=1, \ldots, n$ :

$$
2 a_{i i}>\sum_{j \neq i}\left|a_{i j}+a_{j i}\right|
$$


It is not difficult to see that $a_{i i}$ is always positive for each $i$. Although the condition (22) need not always hold, we can verify that it holds, for example, when $n<4$, $p_{1}=\cdots=p_{n}$ and $c_{1}+k_{1}=\cdots=c_{n}+k_{n}$.

\section{The Nash Equilibrium for Equally Priced Suppliers}

In this section, we study properties, including the existence and uniqueness together with the representation, of the Nash equilibrium of the leader-follower game discussed in the last section. We assume that the prices are all equal, i.e., $p_{1}=\cdots=p_{n} \equiv p$, and also explore how the suppliers compete by changing their delivery frequencies. Under this assumption, we can delete the shared constraint in each supplier's problem (14), since it is automatically satisfied.

For ease of mathematical exposition, we allow (in theory) $r_{i}$ 's to be nonnegative rather than assuming them to be strictly positive. The only requirement is that $r_{1}+$ $\cdots+r_{n} \neq 0$. As we will see below, this additional condition allows us to relax the constraint $l_{i} \leq r_{i} \leq u_{i}$ to simply $r_{i} \geq 0, r \neq 0$, while still preserving the existence and uniqueness of the Nash equilibrium. Then, each supplier's problem (14) is substantially simplified to

$$
\max \phi_{i}\left(r_{i}, r_{-i}\right)=\frac{\alpha_{i} r_{i}}{\sum_{j=1}^{n} r_{j}}-r_{i} \quad \text { s.t. } r_{i} \geq 0,
$$

where $\alpha_{i}$ is defined by (13). This is no longer a generalized Nash game, but an ordinary Nash game. It will be convenient to use the following shorthand notations:

$$
\sigma_{i} \equiv \sum_{\substack{j=1 \\ j \neq i}}^{n} r_{j},
$$

which is the sum of all components of $r$ except $r_{i}$.

4.1 Explicit formula for the unique Nash equilibrium

Lemma 1 For any given $h>0$ and $p>0$, the optimal solution $\lambda=\left(\lambda_{1}, \ldots, \lambda_{n}\right)^{T}$ of the manufacturer's problem (4) is uniquely given by

$$
\lambda_{i}=\frac{r_{i}}{\sum_{j=1}^{n} r_{j}} \geq 0, \quad i=1, \ldots, n
$$

with $\lambda \neq 0$.

Proof. Since $p_{1}=\cdots=p_{n}=p$, it is easy to verify that the unique solution to (9) is given by

$$
v^{*}=\frac{h}{\sum_{j=1}^{n} r_{j}}+p>0 .
$$

Note that we must have

$$
v^{*}-p=\frac{h}{\sum_{j=1}^{n} r_{j}}>0,
$$

since otherwise we have a contradiction to (9). Therefore, by (10), the optimal allocation of the suppliers' shares is given by $(24)$. 
Remark 1 Contrary to our intuition of "giving all contracts to the most frequent supplier", the solution (24) suggests that any supplier with $r_{i}>0$ should have a positive share of the market, no matter how small its frequency is. Practically, it suggests a diversification strategy.

Lemma 2 For any given $r \geq 0$, if $\alpha_{i}>\sigma_{i}$, then there exists $r_{i}^{\prime}>0$ such that $\phi_{i}\left(r^{\prime}\right)>$ 0 , where $r^{\prime}:=\left(r_{1}, \ldots, r_{i-1}, r_{i}^{\prime}, r_{i+1}, \ldots, r_{n}\right)^{T}$.

Proof. Note that

$$
\phi_{i}(r)=\alpha_{i}\left(1-\frac{\sigma_{i}}{r_{i}+\sigma_{i}}\right)-r_{i}
$$

It follows that

$$
\begin{aligned}
\left(r_{i}+\sigma_{i}\right) \phi_{i}(r) & =\alpha_{i}\left(r_{i}+\sigma_{i}-\sigma_{i}\right)-r_{i}\left(r_{i}+\sigma_{i}\right) \\
& =\left(\alpha_{i}-\sigma_{i}\right) r_{i}-r_{i}^{2} .
\end{aligned}
$$

Since $\alpha_{i}-\sigma_{i}>0$ by assumption, there exists an $r_{i}^{\prime}$ sufficiently small and positive such that

$$
\left(\alpha_{i}-\sigma_{i}\right) r_{i}^{\prime}-{r_{i}^{\prime}}^{2}>0
$$

which implies that $\phi_{i}\left(r^{\prime}\right)>0$.

Remark 2 In practice, Lemma 2 says each supplier will be able to turn to profit by just adjusting its delivery frequency. It is reasonable for otherwise this supplier will have no incentive to play the game. In fact, if $\phi_{i}(r)>0$ already, we have $r_{i}>0$ and can simply take $r_{i}^{\prime}=r_{i}$, whereas if $\phi_{i}(r)=0$, there must hold $r_{i}^{\prime} \neq r_{i}$.

With help of Lemma 2, we derive a necessary and sufficient condition concerning the uniqueness of the Nash equilibrium, which is one of main results of this paper.

Theorem 1 The game (23) has a unique positive Nash equilibrium

$$
r_{i}=(n-1)\left(\sum_{j=1}^{n} \alpha_{j}^{-1}\right)^{-1}-\alpha_{i}^{-1}(n-1)^{2}\left(\sum_{j=1}^{n} \alpha_{j}^{-1}\right)^{-2}, \quad i=1, \ldots, n
$$

if and only if

$$
\alpha_{i}>\sigma_{i}, \quad i=1, \ldots, n
$$

Proof. Note that for any fixed $r_{-i}=\left(r_{1}, \ldots, r_{i-1}, r_{i+1}, \ldots, r_{n}\right)^{T}$, the function $\phi_{i}(r)$ is strictly concave in $r_{i}$, which can be seen by the fact that $\partial^{2} \phi_{i} / \partial r_{i}^{2}<0$.

Necessity. If $r>0$ is the Nash equilibrium, then one must have

$$
\frac{\partial \phi_{i}}{\partial r_{i}}=0 \Longleftrightarrow \frac{\alpha_{i} \sigma_{i}}{\left(r_{i}+\sigma_{i}\right)^{2}}-1=0 \Longleftrightarrow r_{i}^{2}+2 \sigma_{i} r_{i}+\sigma_{i}^{2}-\alpha_{i} \sigma_{i}=0 .
$$

For fixed $\sigma_{i}$, the last equation above has a real positive root $r_{i}$ only if $\alpha_{i}>\sigma_{i}$.

Sufficiency. Suppose (27) holds. We claim that at a Nash equilibrium, we have $r_{i}>0$ for all $i$. Indeed, if $r_{i}=0$, one has $\phi_{i}(r)=0$. By Lemma 2 , there exists $r_{i}^{\prime}>0$ such that $\phi_{i}\left(r_{i}^{\prime}, r_{-i}\right)>0$. Therefore, by unilaterally increasing $r_{i}$, player $i$ has an advantage. This conflicts with the definition of a Nash equilibrium. It follows that a Nash equilibrium, if 
exists at all, must be strictly positive. We next prove that the Nash equilibrium exists and is unique since it is the unique positive solution of the equation system

$$
\frac{\partial \phi_{i}}{\partial r_{i}}=0 \Longleftrightarrow\left(\sum_{j=1}^{n} r_{j}\right)^{2}=\alpha_{i} \sigma_{i}, \quad i=1, \ldots, n .
$$

Setting $c \equiv \alpha_{i} \sigma_{i}$, we have $\sigma_{i}=c \alpha_{i}^{-1}$, where $c$ is independent of $i$ since $c=\left(\sum_{j=1}^{n} r_{j}\right)^{2}$ by (28). Then

$$
c \sum_{j=1}^{n} \alpha_{j}^{-1}=\sum_{j=1}^{n} \sigma_{j}=(n-1) \sum_{j=1}^{n} r_{j}=(n-1) \sqrt{c}
$$

which yields

$$
\sum_{j=1}^{n} r_{j}=\sqrt{c}=(n-1)\left(\sum_{j=1}^{n} \alpha_{j}^{-1}\right)^{-1}
$$

while by the definition of $c$ and $\sigma_{i}$,

$$
\alpha_{i} \sum_{\substack{j=1 \\ j \neq i}}^{n} r_{j}=c=(n-1)^{2}\left(\sum_{j=1}^{n} \alpha_{j}^{-1}\right)^{-2}
$$

Multiplying (30) by $\alpha_{i}^{-1}$ and subtracting it from (29), we have

$$
r_{i}=(n-1)\left(\sum_{j=1}^{n} \alpha_{j}^{-1}\right)^{-1}-\alpha_{i}^{-1}(n-1)^{2}\left(\sum_{j=1}^{n} \alpha_{j}^{-1}\right)^{-2}
$$

as desired.

Remark 3 From (30), we have $\sigma_{i}=\alpha_{i}^{-1}(n-1)^{2}\left(\sum_{j=1}^{n} \alpha_{j}^{-1}\right)^{-2}$. Hence the condition (27) can be rewritten as

$$
\alpha_{i}^{2}>(n-1)^{2}\left(\sum_{j=1}^{n} \alpha_{j}^{-1}\right)^{-2}, \quad i=1, \ldots, n
$$

Consider the special case that $\alpha_{1}=\cdots=\alpha_{n}=\alpha$. Then the condition becomes

$$
1>\left(\frac{n-1}{n}\right)^{2}
$$

This is valid and hence implies that condition (31) is not an empty condition. Furthermore, it actually specifies a certain "cost structure" of the suppliers in order for them to stay in business in practice. 


\subsection{Local uniqueness of Nash equilibrium}

We next discuss the question as to when the Nash equilibrium is an isolate point, or in other words, what condition will guarantee that the Nash equilibrium is locally unique. We first define some notations for simplicity of description. Let

$$
E=\left(\begin{array}{cccc}
1 & 1 & \cdots & 1 \\
1 & 1 & \cdots & 1 \\
\cdots & \cdots & \cdots & \cdots \\
1 & 1 & \cdots & 1
\end{array}\right) \text { and } A=\left(\begin{array}{cccc}
0 & -\alpha_{1} & \cdots & -\alpha_{1} \\
-\alpha_{2} & 0 & \cdots & -\alpha_{2} \\
\cdots & \cdots & \cdots & \cdots \\
-\alpha_{n} & -\alpha_{n} & \cdots & 0
\end{array}\right)
$$

Note that, by definition, $A$ is a non-positive matrix in the sense that all entries of the matrix are non-positive.

Applying basic operations, rewrite the Nash equilibrium system (28) as

$$
\psi(r)=0
$$

where

$$
\psi(r) \equiv\left(\begin{array}{c}
\left(e^{T} r\right)^{2}-\left(e^{T} r-r_{1}\right) \alpha_{1} \\
\left(e^{T} r\right)^{2}-\left(e^{T} r-r_{2}\right) \alpha_{2} \\
\cdots \\
\left(e^{T} r\right)^{2}-\left(e^{T} r-r_{n}\right) \alpha_{n}
\end{array}\right)
$$

and $e=(1, \ldots, 1)^{T}$. By direct calculation, the Jacobian matrix of $\psi(r)$ can be expressed as

$$
\psi^{\prime}(r)=\left(2 e^{T} r\right) E+A .
$$

Then, we have the following result immediately.

Proposition 1 Let $r^{*}$ satisfy equation (28). If the Jacobian matrix $\psi^{\prime}\left(r^{*}\right)=\left(2 e^{T} r^{*}\right) E+$ $A$ is nonsingular, then $r^{*}$ is a locally unique solution.

Proof. Since $\psi$ is continuously differentiable in $r$ on some neighborhood of $r^{*}$, the conclusion follows from a classical result in calculus.

According to Proposition 1, we derive the local uniqueness of a Nash equilibrium as follows.

Proposition 2 Let $r^{*}$ be a Nash equilibrium of the game (23). Then it is a locally unique equilibrium.

Proof. By virtue of Proposition 1, we only need to show the Jacobian $\psi^{\prime}\left(r^{*}\right)$ is nonsingular. Recall that $\alpha_{i}, i=1, \ldots, n$, defined by (13) are all positive by the assumption $p>c_{i}+k_{i}$ made in Section 2. Note that $\psi^{\prime}\left(r^{*}\right)$ can be written as

$$
\psi^{\prime}\left(r^{*}\right)=\left(2 e^{T} r^{*}\right) E+A=\left(\begin{array}{cccc}
\delta^{*} & \delta^{*}-\alpha_{1} & \cdots & \delta^{*}-\alpha_{1} \\
\delta^{*}-\alpha_{2} & \delta^{*} & \cdots & \delta^{*}-\alpha_{2} \\
\cdots & \cdots & \cdots & \cdots \\
\delta^{*}-\alpha_{n} & \delta^{*}-\alpha_{n} & \cdots & \delta^{*}
\end{array}\right),
$$

where $\delta^{*} \equiv 2\left(r_{1}^{*}+\cdots+r_{n}^{*}\right)$. Then, by applying some basic operations in linear algebra, we have

$$
\operatorname{det}\left(\psi^{\prime}\left(r^{*}\right)\right)=\operatorname{det}\left(\begin{array}{cc}
M & \varpi \\
\varrho^{T} & \delta^{*}
\end{array}\right)
$$


where $\operatorname{det}(S)$ represents the determinant of a square matrix $S$ and

$$
M \equiv\left(\begin{array}{cccc}
\alpha_{1} & 0 & \cdots & 0 \\
0 & \alpha_{2} & \cdots & 0 \\
\cdots & \cdots & \cdots & \cdots \\
0 & 0 & \cdots & \alpha_{n-1}
\end{array}\right), \quad \varpi \equiv\left(\begin{array}{c}
\delta^{*}-\alpha_{1} \\
\delta^{*}-\alpha_{2} \\
\vdots \\
\delta^{*}-\alpha_{n-1}
\end{array}\right), \quad \varrho \equiv\left(\begin{array}{c}
-\alpha_{n} \\
-\alpha_{n} \\
\vdots \\
-\alpha_{n}
\end{array}\right)
$$

Since the diagonal matrix $M$ is nonsingular, we have

$$
\begin{aligned}
\operatorname{det}\left(\begin{array}{cc}
M & \varpi \\
\varrho^{T} & \delta^{*}
\end{array}\right) & =\operatorname{det}(M) \operatorname{det}\left(\delta^{*}-\varrho^{T} M^{-1} \varpi\right) \\
& =\left[\delta^{*}\left(\sum_{i=1}^{n} \alpha_{i}^{-1}\right)-(n-1)\right] \prod_{i=1}^{n} \alpha_{i} .
\end{aligned}
$$

From (29) and the definition of $\delta^{*}$, we have

$$
\delta^{*}=2(n-1)\left(\sum_{j=1}^{n} \alpha_{j}^{-1}\right)^{-1} .
$$

Thus we obtain

$$
\operatorname{det}\left(\begin{array}{cc}
M & \varpi \\
\varrho^{T} & \delta^{*}
\end{array}\right)=(n-1) \prod_{i=1}^{n} \alpha_{i} \neq 0 .
$$

Hence the Jacobian $\psi^{\prime}\left(r^{*}\right)$ is nonsingular.

From Proposition 2, we conclude that as long as the leaders' game has a Nash equilibrium, the equilibrium point will be isolated (locally unique).

\subsection{Discussion}

We continue to consider the case when all the suppliers offer an identical price. Denote

$$
\gamma \equiv(n-1)\left(\sum_{i=1}^{n} \frac{1}{\alpha_{i}}\right)^{-1}
$$

By (29), this is actually equal to the sum of delivery frequencies $r_{i}$ of all suppliers, i.e., $\gamma=\sum_{i=1}^{n} r_{i}$. Moreover, by (26), each supplier's delivery frequency satisfies

$$
r_{i}=\gamma\left(1-\frac{\gamma}{\alpha_{i}}\right), \quad i=1, \ldots, n .
$$

This gives a formula for supplier $i$ to decide its delivery frequency. If the information is incomplete, then supplier $i$ would not know the exact values $\alpha_{j}$ of other suppliers $j \neq i$. However, the supplier could at least have an estimate on $\gamma$ based on market information. Therefore the above formula can be used to determine $r_{i}$ approximately.

Substituting (32) into the objective function $\phi_{i}$ of the game (23) and taking into account the relation $\gamma=\sum_{j=1}^{n} r_{j}$, we obtain the profit of supplier $i$ as

$$
\phi_{i}(r)=\frac{\alpha_{i} r_{i}}{\sum_{j=1}^{n} r_{j}}-r_{i}=\frac{\left(\alpha_{i}-\gamma\right)^{2}}{\alpha_{i}}
$$


Finally, the necessary and sufficient condition $\alpha_{i}>\sigma_{i}$ for the existence of a positive Nash equilibrium shown in Theorem 1 gives rise to the necessary "minimum profit margin" of supplier $i$ to stay at the Nash equilibrium. It says that the frequency game cannot have an equilibrium if any supplier seeks to enlarge its market share by just lowering its selling price. This is somewhat surprising and not intuitive at all. This explains why it is not wise for a supplier to produce at the breakeven price. In fact, the results suggest that firms should look for ways to stabilize their cost structures while at the same time having a flexible manufacturing system that permits changes in delivery frequency.

Proposition 3 Suppose that all suppliers offer the identical price $p_{1}=\cdots=p_{n}=p$. Then, for any $i, j, l \in\{1,2, \ldots, n\}$, we have

$$
\begin{aligned}
0= & \left(\frac{C_{j}+K_{j}}{p-c_{j}-k_{j}}-\frac{C_{l}+K_{l}}{p-c_{l}-k_{l}}\right) r_{i}+\left(\frac{C_{l}+K_{l}}{p-c_{l}-k_{l}}-\frac{C_{i}+K_{i}}{p-c_{i}-k_{i}}\right) r_{j} \\
& +\left(\frac{C_{i}+K_{i}}{p-c_{i}-k_{i}}-\frac{C_{j}+K_{j}}{p-c_{j}-k_{j}}\right) r_{l} .
\end{aligned}
$$

Proof. By (28), it follows that

$$
\alpha_{i}\left(r^{T} e-r_{i}\right)=\alpha_{j}\left(r^{T} e-r_{j}\right)=\alpha_{k}\left(r^{T} e-r_{k}\right) .
$$

Then, we have

$$
\begin{aligned}
& \alpha_{i} \alpha_{j} r_{i}+\alpha_{j} \alpha_{l}\left(r^{T} e-r_{l}\right)=\alpha_{i} \alpha_{j} r_{i}+\alpha_{j} \alpha_{i}\left(r^{T} e-r_{i}\right)=\alpha_{j} \alpha_{i} r^{T} e \\
= & \alpha_{i} \alpha_{j}\left(r^{T} e-r_{j}+r_{j}\right)=\alpha_{i} \alpha_{j} r_{j}+\alpha_{i} \alpha_{j}\left(r^{T} e-r_{j}\right)=\alpha_{i} \alpha_{j} r_{j}+\alpha_{i} \alpha_{l}\left(r^{T} e-r_{l}\right) .
\end{aligned}
$$

Then, it yields that

$$
\begin{array}{r}
\alpha_{i} \alpha_{j} r_{i}+\alpha_{j} \alpha_{l} r_{i}+\alpha_{j} \alpha_{l} r_{j}+\alpha_{j} \alpha_{l}\left(r^{T} e-r_{i}-r_{j}-r_{l}\right) \\
=\alpha_{i} \alpha_{j} r_{j}+\alpha_{i} \alpha_{l} r_{j}+\alpha_{i} \alpha_{l} r_{i}+\alpha_{i} \alpha_{l}\left(r^{T} e-r_{i}-r_{j}-r_{l}\right) .
\end{array}
$$

Applying some basic operations, it follows from the above equation that

$\left(\alpha_{i} \alpha_{j}+\alpha_{j} \alpha_{l}-\alpha_{i} \alpha_{l}\right) r_{i}-\alpha_{l} \alpha_{j} r_{i}-\alpha_{l} \alpha_{j} r_{l}=\left(\alpha_{i} \alpha_{j}+\alpha_{i} \alpha_{l}-\alpha_{j} \alpha_{l}\right) r_{j}-\alpha_{l} \alpha_{i} r_{j}-\alpha_{i} \alpha_{k} r_{l}$

Then, it gives that

$$
\alpha_{i}\left(\alpha_{j}-\alpha_{l}\right) r_{i}=\alpha_{j}\left(\alpha_{i}-\alpha_{l}\right) r_{j}+\alpha_{l}\left(\alpha_{j}-\alpha_{i}\right) r_{l},
$$

which can be written as

$$
\alpha_{i}\left(\alpha_{l}-\alpha_{j}\right) r_{i}+\alpha_{j}\left(\alpha_{i}-\alpha_{l}\right) r_{j}+\alpha_{l}\left(\alpha_{j}-\alpha_{i}\right) r_{l}=0 .
$$

Dividing each item on the left side of the above equation by $\alpha_{i}, \alpha_{j}, \alpha_{l}$, it gives that

$$
\left(\frac{1}{\alpha_{j}}-\frac{1}{\alpha_{l}}\right) r_{i}+\left(\frac{1}{\alpha_{l}}-\frac{1}{\alpha_{i}}\right) r_{j}+\left(\frac{1}{\alpha_{i}}-\frac{1}{\alpha_{j}}\right) r_{l}=0 .
$$

The result follows immediately by replacing each $\alpha_{s}$ with $\frac{p_{s}-c_{s}-k_{s}}{C_{s}+K_{s}}$. This completes the proof.

Proposition 3 describes an interesting relationship between the delivery frequencies and the contribution margins of the underlying suppliers. Note that the sum of the coefficients in equation (33) is equal to zero. Note also that (33) actually reflects a linear relationship among the delivery frequencies, which shows the higher the delivery frequency the higher the profit per unit cost. 


\section{Conclusion}

In this paper, we have examined a delivery frequency competition game of $n$ competing suppliers and one manufacturer. A generalized Nash equilibrium has been considered first under a general setting, and the existence and uniqueness of an equilibrium have been discussed by way of the variational inequality formulation. For the special case of equal selling price, we have derived an explicit formula for a Nash equilibrium and given a necessary and sufficient condition for the existence and uniqueness of such an equilibrium.

Acknowledgements The research is partially supported by Centre for Maritime Studies, The Provost's Chair Grant, and The Logistics Institute - Asia Pacific of National University of Singapore. This work is also supported in part by a Grant-in-Aid for Scientific Research from Japan Society for the Promotion of Science.

\section{References}

1. K.J. Arrow, G. Debreu: Existence of an equilibrium for a competitive economy, Econometrica, 22, 265-290 (1954)

2. S. Benjaafar, E. Elahi, K. L. Donohue: Outsourcing via service competition, Manag. Sci. 53, 241-259 (2007)

3. F. Bernstein, A. Federgruen: Coordination mechanisms for supply chains under price and service competition, Manufac. Serv. Oper. Manag. 9, 242-262 (2007)

4. G. Cachon, P. Harker: Competition and outsourcing with scale economies, Manag. Sci. 48 , 1314-1333 (2002)

5. G. Cachon, F. Zhang: Obtaining fast service in a queueing system via performance-based allocation of demand, Manag. Sci. 53, 408-420 (2007)

6. G. Debreu: A social equilibrium existence theorem, Proc. Natl. Acad. Sci. USA, 38, 886-893 $(1952)$

7. F. Facchinei, C. Kanzow: Generalized Nash equilibrium problems, 4OR 5, 173-210 (2007)

8. F, Facchinei, J.-S. Pang: Finite-Dimensional Variational Inequalities and Complementarity Problems, Springer-Verlag: New York, (2003)

9. A. Federgruen, A. Heching: Combined pricing and inventory control under uncertainty, Oper. Res. 47, 454-475 (1999)

10. J. Hall, E. Porteus: Customer service competition in capacitated systems, Manufac. Serv. Oper. Manag. 2, 144-165 (2000)

11. P.T. Harker, Generalized Nash games and quasi-variational inequalities, European J. Oper. Res. 54, 81-94 (1991)

12. E. Kalai, M. Kamien, M. Rubinovitch: Optimal service speeds in a competitive environment, Manag. Sci., 38, 1154-1163 (1992)

13. L. Li: The role of inventory in delivery-time competition, Manag. Sci. 38, 182-197 (1992)

14. L. Li, Y.S. Lee: Pricing and delivery-time performance in a competitive environment, Manag. Sci. 40, 633-646 (1994)

15. G.H. Lin, M. Fukushima: Stochastic equilibrium problems and stochastic mathematical programs with equilibrium constraints: A survey, Pac. J. Optim. 6, 455-482 (2010)

16. C. Loch, Time competition is capability competition, Working Paper, INSEAD, (1994)

17. J.-S. Pang, M. Fukushima: Quasi-variational inequalities, generalized Nash equilibria, and multi-leader-follower games, Comput. Manag. Sci. 2, 21-56 (2005)

18. J.B. Rosen: Existence and uniqueness of equilibrium points for concave $N$-person games, Econometrica, 38, 520-534 (1965)

19. K. So, J.S. Song: Price, delivery time guarantees and capacity selection, European J. Oper. Res. 111, 28-49 (1998) 